\title{
Formação profissional em Educação Física e saúde na Universidade Federal de São Paulo
}

Rev Bras Ativ Fis Saúde p. 646-651 DOI:

http://dx.doi.org/10.12820/rbafs.v.18n5p646

1 Universidade Federal de São Paulo Campus Baixada Santista, Departamento de Ciências do Movimento Humano, Santos, São Paulo, Brasil.

2 Graduado em Educação Física pela Universidade Federal de São Paulo - Campus Baixada Santista, Santos, São Paulo, Brasil.

3 Universidade Federal de São Paulo Campus Baixada Santista, Departamento de Biociências, Santos, São Paulo, Brasil.

\section{Resumo}

O objetivo deste texto é apresentar o curso de Bacharelado em Educação Física - Modalidade Saúde da Universidade Federal de São Paulo - Campus Baixada Santista. O referido curso iniciou suas atividades em 2006, almejando a formação de um profissional que esteja habilitado às demandas do universo do sistema de saúde. Em sua estrutura curricular, o curso apresenta 4 eixos: $\mathrm{O}$ ser humano em sua dimensão biológica; $\mathrm{O}$ ser humano e sua inserção social; Trabalho em Saúde, e; Aproximação à prática específica de Educação Física. Após formatura de quatro turmas o curso passa por avaliação de seu Projeto Pedagógico, entendendo a necessidade de adequações que permitam fortalecer a formação em Educação Física e saúde na instituição. Contudo, os resultados iniciais suportam a continuidade do projeto pedagógico, o qual se compreende adequado para a formação de um profissional disposto ao diálogo interdisciplinar e ao trabalho interprofissional no sistema de saúde.

\section{Palavras-chave}

Educação Física e Treinamento. Saúde. Formação de Recursos Humanos.

\begin{abstract}
The propose of this paper is to present the course of Bachelor of Physical Education - Health Modality of Federal University of São Paulo - Campus Baixada Santista. This course began activities in 2006, aiming the formation of a professional who is qualified to the demands of the health care's universe. The curriculum of the course has 4 axes: The Human in its Biological Dimension; Human Being and their Social Integration; Health Work, and; Approach to the Specific Practice of Physical Education. After graduation of four classes, the course undergoes to a curricular reevaluation, understanding the need for adjustments for strengthening the formation in Physical Education and Health at the institution. However, the initial results support the continuity of the education program, which it shows suitable to the formation of a professional willing to the interdisciplinary dialogue and to the interprofessional work in the health system.
\end{abstract}

\section{Keywords}

Physical Education and Training. Health. Human Resources Formation. 


\section{INTRODUÇÃO}

A discussão sobre a formação profissional em Educação Física (EF) tem início na década de 1930, na era Vargas¹. Deste período até a década de 1980, várias foram as conquistas que garantiam uma formação mais sólida na área. À exemplo, podemos citar a Constituição de 1937 (EF obrigatória nas escolas), a Lei de Diretrizes e Bases da Educação de 1961 (exigência de um currículo mínimo e um núcleo de disciplinas que pudesse garantir formação cultural e profissional adequadas), criação de um grupo de trabalho no Departamento de EF do Ministério da Educação e Cultura em 1968 (responsável por iniciar uma reforma curricular na EF), dentre outras iniciativas ${ }^{1-2}$.

Contudo, é na década de 1980 que o debate acumulado na área resulta na promulgação de uma Resolução $(03 / 87)^{3}$, a qual buscou padronizar a formação em EF e contemplar 2 modalidades ${ }^{2}$ - bacharelado e licenciatura. Entretanto, o caminho para tal resolução foi fruto mais de divergências do que de consensos, haja vista que foi neste período que a EF brasileira vivenciou sua crise epistemológica. Consequentemente, foi inaugurado um período tenso, conflituoso e de extremas divergências sobre a formação e intervenção em EF, gerando desdobramentos igualmente conflitantes nos anos seguintes.

Na década de 1990, por conta da criação do bacharelado, a discussão centrouse em torno da regulamentação profissional, que, em 1998, por meio da Lei 9696, a EF sai do status de ocupação para o de profissão ${ }^{4}$. Porém, tal legislação não foi concebida e recebida de forma harmônica pela comunidade acadêmica. $V_{a z}^{5}$, ao discutir a questão na época apontando as fragilidades do processo, afirma que se fazia necessário maior amplitude do debate em detrimento aos diálogos mais entrincheirados. Contudo, em que pese às divergências existentes, que, por limites desse texto, não permitem maiores desdobramentos, é pertinente ressaltar que a mesma acabou por interferir nas discussões sobre a formação inicial, principalmente no que se refere à formação do bacharel que, apesar de ser prevista desde 1987, passa a ser discutida com mais ênfase neste período (final do século XX e início do século XXI), culminando na Resolução $7 / 2004^{6}$, que estabelece as Diretrizes para a formação em EF. Assim, vários cursos no Brasil se reestruturaram para atender essa demanda, a exemplo da Faculdade de EF da Unicamp ${ }^{7}$.

Contudo, a relação entre a formação profissional em EF e a área da saúde constituiu-se de forma frágil, haja vista os estudos que apontam inconsistências na formação profissional em EF considerando a atuação nos serviços de saúde ${ }^{8-10}$. Cabe ressaltar que, neste texto, o termo "serviços de saúde" significará, apesar da existência de outras correspondências, toda e qualquer área de atuação que se relacione com a saúde das pessoas. Ou seja, para além do Sistema Único de Saúde (SUS), que, no caso da EF, também pode abranger, como exemplo: academias, clínicas especializadas, centros de reabilitação, spas, dentre outros.

Dessa forma, é que este trabalho apresenta o curso de EF da Universidade Federal de São Paulo - Campus Baixada Santista (UNIFESP-BS), o qual tem sido referenciado como uma importante iniciativa para responder as demandas da formação em EF para a saúde ${ }^{11-12}$.

\section{Breve histórico do curso}

A UNIFESP-BS foi criada em 2005, sendo que em dezembro do mesmo ano aconteceu o primeiro vestibular para os cursos de EF, Fisioterapia, Nutrição, Psicologia e Terapia Ocupacional. Recentemente, o curso de Serviço Social foi 
incorporado ao campus. De acordo com o Projeto Político Pedagógico do Campus (PPP) ${ }^{13}$, o Plano de Desenvolvimento Institucional de 2005 assumia as seguintes metas:

- Criação de novos cursos de Graduação, em áreas contiguas a da saúde;

- Continuidade e fortalecimento da iniciativa de oferta de novas modalidades de cursos que complementam o Ensino Médio;

- Expansão da Graduação para novas áreas do conhecimento - uma nova metadisciplinaridade;

- Expansão do escopo geográfico da UNIFESP - novos campi.

Assim, desde sua implantação, a UNIFESP-BS enfatiza o trabalho em saúde como base para todos os seus cursos, priorizando uma linha de cuidado que, historicamente, a instituição se inscreve.

\section{O curso de Educação Física da UNIFESP-BS}

O curso de Bacharelado em EF - Modalidade Saúde - da UNIFESP-BS tem duração mínima de 4 e máxima de 6 anos, possuindo, atualmente, a carga horária total de 4420 horas cursadas em período integral. O mesmo se orienta pelos seguintes princípios: prática profissional como eixo pedagógico norteador; problematização do ensino a partir da prática e pesquisa; interdisciplinaridade (integração das disciplinas); interprofissionalismo (trabalho em equipe); postura ativa na construção do conhecimento; postura mediadora do docente no processo ensino-aprendizagem; integração com a comunidade, e; integração dos diferentes níveis de ensino e pesquisa ${ }^{14}$.

Assim propõe-se como primordial a produção de um trabalho integrado, preconizando uma intervenção voltada à interação entre os fenômenos biológicos, humanos e sociais objetivando uma visão de saúde integral da população ${ }^{14}$

Para tanto, a formação é estruturada em 4 eixos (Figura 1) que transversam toda a graduação. São 3 eixos comuns à todos os cursos e 1 eixo específico para cada curso, a saber:

- O ser humano e sua Inserção social (IS);

- Trabalho em Saúde (TS);

- O ser humano e sua dimensão biológica (BIO);

- Aproximação à prática especifica de EF (ESPECÍFICO).

O eixo IS preconiza atividades de ensino que considere o impacto da noção de cultura sobre a concepção de ser humano, além de instrumentalizar os alunos para o relacionamento pessoal com os usuários e profissionais dos serviços de saúde ${ }^{13-14}$. Almeja ainda sensibilizar o aluno para sua função educativa no futuro exercício da profissão ${ }^{13-14}$.

O eixo TS aborda a problematização das múltiplas dimensões envolvidas no processo saúde-doença e de produção de cuidados ${ }^{13-14}$. Ainda, visa compreender os principais problemas de saúde da população, propiciar conhecimento sobre o SUS, compreender o processo de trabalho em saúde, bem como contribuir para o desenvolvimento de competências para o trabalho em equipe, para o diálogo interprofissional $1^{13-14}$.

O eixo BIO busca propiciar o entendimento do funcionamento e da interação entre os diferentes sistemas do organismo, caracterizando suas bases celula- 
res e moleculares e habilitando os profissionais em formação a discutir de forma abrangente e interdisciplinar a relevância dos processos biológicos nas diferentes doenças ${ }^{13-14}$.

Já o eixo ESPECÍFICO busca, desde o início do curso, oferecer uma formação que concebe o exercício físico/práticas corporais como estratégia terapêutica não medicamentosa para intervenção na área da saúde, baseada em prescrição adequada e individualizada às demandas das pessoas ${ }^{14}$. Neste contexto, são desenvolvidos conteúdos e atividades sistematizadas para intervir em serviços de saúde, visando a inserção dos futuros profissionais em equipes interprofissionais de diferentes locais de trabalho voltados a promoção da saúde e da qualidade de vida, bem como o controle de doenças crônicas ${ }^{14}$. Este eixo é desenvolvido por meio de 6 sub-eixos: Aproximação à prática da EF (APROX); Fundamentos das atividades físicas e esportivas (FAFES); Estudo do Movimento Humano (EMH); Ciências aplicadas à EF (CAEF); Ciências do exercício físico (CEF) e Produção de Conhecimento em EF e saúde (PCEF) (Figura 2).

Ainda existem as Atividades Complementares de Graduação (ACG) (240hs) e os Módulos Eletivos (ELET) (120hs). Os Módulos Eletivos são oferecidos ao longo do curso de graduação de EF. Cada módulo tem, no mínimo, 40hs e máximo de 80 hs. Os mesmos são oferecidos na perspectiva de uma experiência acadêmica complementar, promovendo enriquecimento cultural, aprofundamento e/ou atualização de conhecimentos, possibilitando ao aluno a flexibilização de sua formação.

Em síntese, a formação profissional em EF e saúde na UNIFESP-BS é assim disposta:

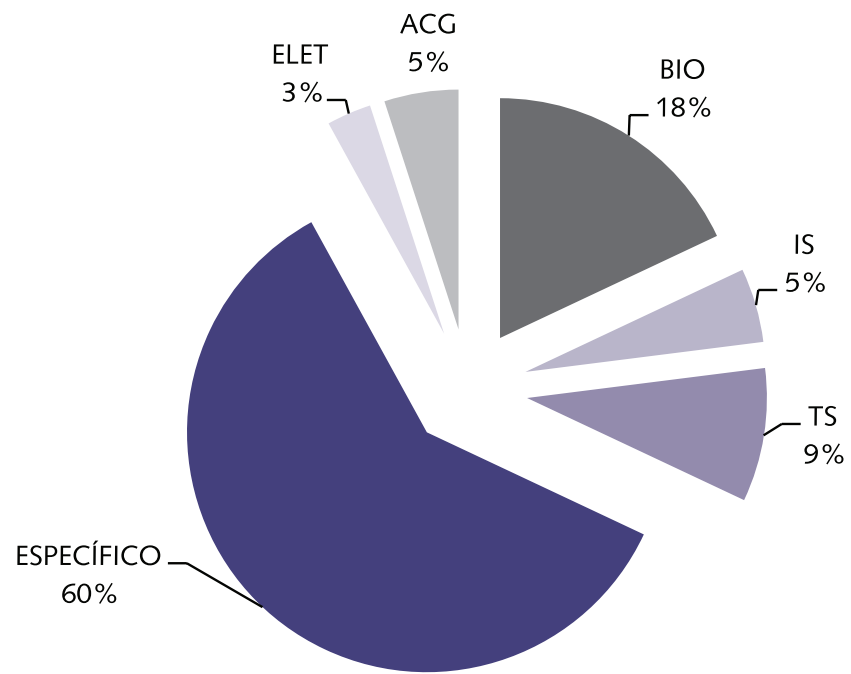

Figura 1 - Gráfico da distribuição da carga horária total do curso. BIO - Eixo "O Ser humano e sua dimensão biológica"; IS - Eixo "O ser humano e sua inserção social"; TS - Eixo "Trabalho em Saúde"; ESPECíFICO - Eixo "Aproximação à prática específica de Educação Física"; ELET - Módulos Eletivos; ACG - Atividades complementares de graduação (ACG).

Desse modo, a formação do Bacharel em EF - Modalidade Saúde - pela UNIFESP-BS objetiva preparar o aluno à: diagnosticar, planejar, prescrever, orientar, assessorar, supervisionar, controlar e avaliar projetos e programas de atividades físicas, recreativas e esportivas nas perspectivas da prevenção, promoção, proteção e reabilitação da saúde. Assim, visa garantir uma formação específica conforme os princípios norteadores das Resoluções 7/20046 e 4/200915. 


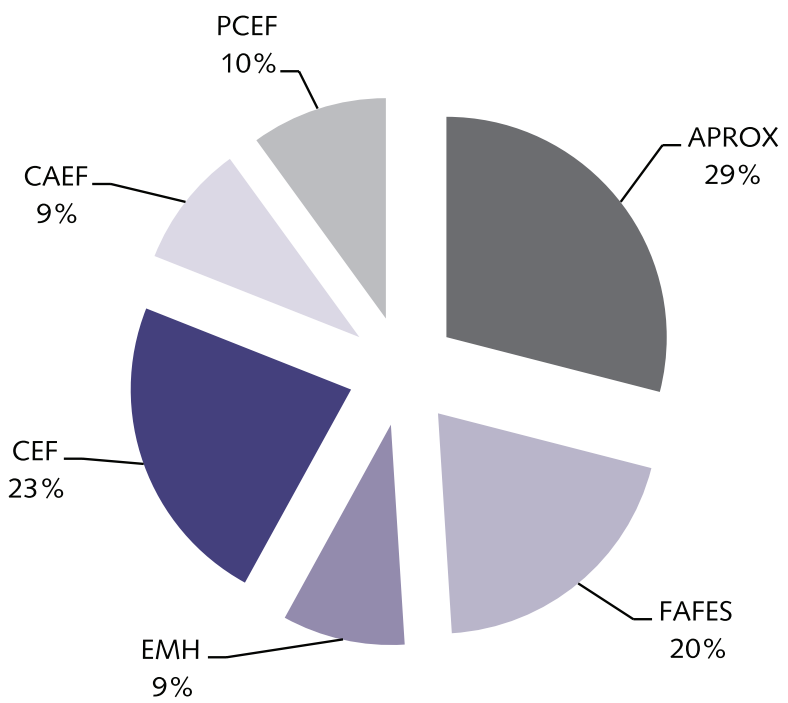

Figura 2 - Gráfico da distribuição da carga horária do eixo específico. APROX - Aproximação à prática específica da Educação Física; FAFES - Fundamentos das atividades físicas e esportivas; EMH - Estudo do movimento humano; CEF - Ciências do exercício físico; CAEF - Ciências aplicadas à Educação Física; PCEF - Produção de conhecimento em Educação Física.

\section{CONCLUSÃO}

O Curso iniciou suas atividades em 2006, almejando uma formação profissional que habilite o egresso às demandas do sistema de saúde, de forma ética e humana. Isso porque, as transformações sociais exigem que a Universidade crie cenários e propostas pedagógicas incorporando a realidade contextual na formação profissional. Após a formatura de 4 turmas, sendo notada evasão na ordem de $20 \%$ e inserção dos egressos, principalmente, no contexto do sistema de saúde e pós graduação stricto sensu, o curso passa por avaliação de seu Projeto Pedagógico, se atendo aos seguintes aspectos: flexibilização curricular; EF no contexto hospitalar; integração entre ensino e extensão, e; tempo de integralização. O elemento fomentador dessa avaliação é fruto de agenda ampliada para (re)discussão do PPP da instituição como um todo, haja vista a existência de um cenário institucional aberto ao permanente debate da formação em saúde. Pretende-se, até o final de 2014, consolidar as ações já existentes e implantar as que se apresentam como possibilidades pertinentes à formação em EF e saúde.

Entretanto, os resultados iniciais suportam a continuidade do Projeto Pedagógico, o qual consideramos se aproximar do ideal para a formação de um profissional de EF autônomo, crítico, criativo e disposto ao diálogo interdisciplinar e o trabalho interprofissional no sistema de saúde.

\section{Contribuição dos autores}

Ferreira SE, Guerra RLF, Pacheco PFL foram responsáveis pela concepção, redação e revisão crítica do trabalho. Gomes RJ, Azevedo PHSM, Botero JP foram responsáveis pela redação e revisão crítica do trabalho. Oliveira RC foi responsável pela concepção, redação, revisão crítica do trabalho e aprovação da versão a ser submetida.

\section{REFERÊNCIAS}

1. Souza Neto S, Alegre AN, Hunger D, Pereira JM. A formação do profissional de educação física no Brasil: uma história sob a perspectiva da legislação federal no século XX. Rev Bras Ciênc Esporte. 2004; 25 (2): 113-28. 
2. Azevedo ACB, Malina A. Memória do currículo de formação profissional em educação física no Brasil. Rev Bras Ciênc Esporte. 2004; 25 (2): 129-42.

3. Brasil. Conselho Federal de Educação. Resolução n.3, 16 de junho de 1987. Diário Oficial n.172, Brasília, 1987.

4. Benites LC, Souza Neto S, Hunger D. O processo de constituição histórica das diretrizes curriculares na formação de professores de Educação Física. Educ Pesq. 2008; 34 (2): 343-60.

5. Vaz A. Regulamentação da profissão: desejo e mal-estares. Movimento 2011; 7 (4): 20-27.

6. Brasil. Conselho Nacional de Educação. Resolução CNE/CES n.07, de 31 de março de 2004. Institui as Diretrizes Curriculares Nacionais para os cursos de graduação em Educação Física, em nível superior de graduação plena. Brasília: Diário Oficial da União, 05 Abr. 2004, Seção 1, p.18.

7. Chacon-Mikail MPT, Montagner PC, Madruga VA. Educação Física: formação acadêmica e atuação profissional no campo da saúde. Motriz. 2009; 15 (1): 192-8.

8. Anjos TC, Duarte ACGO. A educação física e a estratégia de saúde da família: formação e atuação profissional. Physis. 2009; 19 (4): 1127-43.

9. Brugnerotto F, Simões R. Caracterização dos currículos de formação profissional em Educação Física: um enfoque sobre saúde. Physis. 2009; 19 (1): 149-72.

10. Pasquim HM. A saúde coletiva nos cursos de graduação em educação física. Saúde soc. 2010; 19 (1): 193-200.

11. Fonseca SA, Menezes AS, Feitosa WNM, Loch MR. Notas preliminares sobre a Associação Brasileira de Ensino da Educação Física para a saúde - Abenefs. Cad Fnepas. 2012; 2: 38-48.

12. Fonseca SA, Menezes AS, Loch MR, Feitosa WNM, Nahas MV, Nascimento JV. Pela criação da Associação Brasileira de Ensino da Educação Física para a Saúde: Abenefs. Rev Bras Ativ Fis e Saúde. 2011; 16(4): 283-8.

13. Unifesp. Campus Baixada Santista. Projeto Político Pedagógico, 2005.

14. Unifesp. Campus Baixada Santista. Projeto Político Pedagógico: Curso de Educação Física, 2009.

15. Brasil. Conselho Nacional de Educação. Resolução CNE/CES n.04, de 6 de abril de 2009. Dispõe sobre carga horária mínima e procedimentos relativos à integralização e duração dos cursos de graduação em Biomedicina, Ciências Biológicas, Educação Física, Enfermagem, Farmácia, Fisioterapia, Fonoaudiologia, Nutrição e Terapia Ocupacional, bacharelados, na modalidade presencial. Brasília: Diário Oficial da União, 11 Mar. 2009.

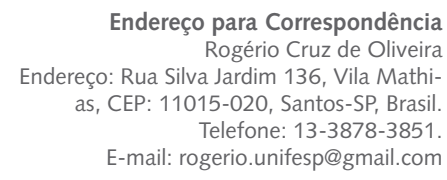

Recebido 22/07/2013

Revisado 13/11/2013

$21 / 11 / 2013$

Aprovado 04/12/2013 